\title{
Exact Ground State Density-Functional Theory for Impurity Models Coupled to External Reservoirs and Transport Calculations
}

\author{
Peter Schmitteckert ${ }^{1}$ and Ferdinand Evers ${ }^{1,2}$ \\ ${ }^{1}$ Institut für Nanotechnologie, Forschungszentrum Karlsruhe, D-76021 Karlsruhe, Germany \\ ${ }^{2}$ Institut für Theorie der Kondensierten Materie, Universität Karlsruhe, D-76128 Karlsruhe, Germany
}

(Received 19 June 2007; published 28 February 2008)

\begin{abstract}
A method is presented employing the density matrix renormalization group to construct exact ground state (GS) exchange correlation functionals for models of correlated electrons coupled to leads. We apply it to show that conductance calculations with Kohn-Sham GS density-functional theory can yield quantitative results in the Coulomb blockade regime. Our study is relevant for "molecular electronics" since it strongly suggests that the well documented discrepancies between theoretical and experimental transport coefficients originate (mainly) from approximations in GS functionals.
\end{abstract}

DOI: 10.1103/PhysRevLett.100.086401

PACS numbers: 71.15.Mb, 05.45.Df, 72.15.Rn

Running an electrical current through individual molecules and being able to control the current flow by molecular design is one of the intriguing aspects of molecular electronics. The interesting behavior of "molecular devices" is typically related to specific properties of the molecule at hand. For a detailed understanding of experiments on single molecules, e.g., the current voltage characteristics, $a b$ initio calculations for transport properties are indispensable. The particular challenge of $a b$ initio calculations for transport quantities is to accurately describe wave function hybridization with the contacts. To this end, one needs to treat a sizable part of the contact on the same footing as the molecule. Sufficient system sizes are achieved with the current "standard method", which is a combination of Kohn-Sham (KS) density-functional theory (DFT) calculations and the (self consistent) Landauer approach [1-3]. At least for larger molecules, comprising a few hundred electrons, the standard approach appears to be without computationally feasible alternative, at present.

An exact relation between the linear current density $\mathbf{j}$, the KS-Kubo-conductivity and the exchange correlation (XC) functional of time dependent DFT, $\mathbf{E}_{\mathrm{XC}}(\mathbf{r}, \omega)$, has been derived in Ref. [4],

$\mathbf{j}(\mathbf{r}, \omega)=\int d^{3} r^{\prime} \hat{\sigma}_{S}\left[n_{0}\right]\left(\mathbf{r}, \mathbf{r}^{\prime}, \omega\right)\left[\mathbf{E}_{\mathrm{tot}}\left(\mathbf{r}^{\prime}, \omega\right)+\mathbf{E}_{\mathrm{XC}}\left(\mathbf{r}^{\prime}, \omega\right)\right]$.

$\left[\mathbf{E}_{\mathrm{tot}}(\mathbf{r}, \omega)\right.$ denotes the sum of external and Hartree field, $n_{0}(\mathbf{r})$ the ground state electron density.] In one dimension and the dc-limit, $j(\mathbf{r}, \omega \rightarrow 0)=I$, so Eq. (1) reduces to $I=$ $g_{S}\left(V_{\text {tot }}+V_{\mathrm{XC}}\right)$ introducing the KS-conductance, $g_{S}$, the physical voltage $V_{\text {tot }}$ and its XC-shift $V_{\mathrm{XC}}$ [4].

Within the framework of Eq. (1), the approximations underlying virtually all present-days application of the standard method for conductance calculations can be analyzed. (i) Dynamical corrections $V_{\mathrm{XC}}$ or $\mathbf{E}_{\mathrm{XC}}$, are universally ignored even though $\mathbf{E}_{\mathrm{XC}}(\omega)$ is generally important for the calculation of excitation energies [5]. (ii) The XC functional, which should be used in quasistatic nonequilibrium to calculate $g_{s}$, is replaced by an approximate ground state functional, usually in a local density approximation. The quantitative impact of (i) is not known at present. In contrast, (ii) must be expected to have severe consequences, since the missing derivative discontinuity in available XC functionals can artificially boost the DFT level broadening $\Gamma_{\mathrm{DFT}}$ observed in the variation of $g_{S}$ with the gate voltage, $V_{\text {gate }}$, from its true value $\Gamma$ up to the interaction energy $U \gg \Gamma$. Thus, an accurate prediction of transport coefficients is extremely difficult. In practice usually large quantitative deviations, typically an order of magnitude, plague the comparison between theoretical and experimental conductances $[4,6,7]$.

The purpose of the present work is twofold. First, we investigate a model system for a correlated quantum dot, the $M$-site interacting resonant level model (IRLM), for which we can show that the dynamical corrections (i) are in fact small. We give an argument, why we expect validity of this result for a broader class of dots including molecules. This aspect of our work is important good news because it justifies the neglect of dynamical correlations and hence the employment of a (relatively simple) single particle scattering approach to the conductance, which is inherent to the standard approach.

Second we propose and apply a novel method which is based on the marriage of DFT with the density matrix renormalization group (DMRG) technique to achieve our first goal. The DFT and DMRG approach allows us to construct numerically exact ground state (GS) functionals for a broad class of generic model systems of correlated fermions; its principle has been introduced before by Gunnarsson and Schönhammer [8] and with an application to a one-dimensional Hubbard chain by Schönhammer, Gunnarsson, and Noack [9]. The crucial extension proposed in this Letter is, that our systems are not homogeneous leading to spatially varying Kohn-Sham potentials. Since our leads provide a fermionic bath, the method 
should be generally useful to investigate fundamental properties of XC potentials that describe correlated electrons coupled to a thermodynamic bath of free fermions. Here, we apply it to obtain the exact KS conductance $g_{S}$ for the IRLM. By comparing to the exact physical conductance, $g=I / V_{\text {tot }}$, it is demonstrated that $g_{S}$ gives a highly accurate estimate for position and broadening of transport resonances even in the Coulomb blockade regime. In this sense, dynamical corrections $V_{\mathrm{XC}}$, including, e.g., a viscous part [10], are small.

The Hamiltonian of the $M$-site IRLM model reads $\mathcal{H}=$ $\mathcal{H}_{e \mathcal{M}}+\mathcal{H}_{U}+\mathcal{H}_{\mathcal{R}}+\mathcal{H}_{T}$, with

$$
\mathcal{H}_{e \mathcal{M}}=V_{\text {gate }} \sum_{\ell \in \mathcal{M}} \hat{c}_{\ell}^{\dagger} \hat{c}_{\ell}-\sum_{\ell, \ell-1 \in e \mathcal{M}}\left(t_{\ell} \hat{c}_{\ell}^{\dagger} \hat{c}_{\ell-1}+\text { H.c. }\right)
$$

$$
\mathcal{H}_{U}=U \sum_{\ell, \ell-1 \in \mathcal{M}}\left(\hat{n}_{\ell}-\frac{1}{2}\right)\left(\hat{n}_{\ell-1}-\frac{1}{2}\right)
$$

where $\hat{c}_{\ell}^{\dagger}\left(\hat{c}_{k}^{\dagger}\right)$ and $\hat{c}_{\ell}\left(\hat{c}_{k}\right)$ are spinless fermionic creation and annihilation operators at site $\ell$ (at momentum $k$ ), $\hat{n}_{\ell}=$ $\hat{c}_{\ell}^{\dagger} \hat{c}_{\ell}$. Furthermore, $\mathcal{H}_{\mathcal{R}}=\sum_{k \in \mathcal{R}_{L}, \mathcal{R}_{R}} \epsilon_{k} \hat{c}_{k}^{\dagger} \hat{c}_{k}$ and

$$
\mathcal{H}_{T}=-\left(\sum_{k \in \mathcal{R}_{L}} t_{k} \hat{c}_{k}^{\dagger} \hat{c}_{1}+\sum_{k \in \mathcal{R}_{R}} t_{k} \hat{c}_{k}^{\dagger} \hat{c}_{M_{E}}\right)+\text { H.c. }
$$

Indices denote Hilbert spaces of the molecule proper (nanostructure or quantum dot), $\mathcal{M}$, of the extended molecule, $e \mathcal{M}$ and of the left and right electrode reservoirs, $\mathcal{R}_{L}, \mathcal{R}_{R} . \mathcal{H}_{T}$ denotes the tunneling Hamiltonian describing the contact between reservoirs and the extended molecule; the interaction on the molecule is modeled by $\mathcal{H}_{U}$. ( $U=2 t$ in all calculations presented.) The indices 1 and $M_{E}$ denote the first and last site in $e \mathcal{M}$. The general setup is displayed in Fig. 1. For the single level model the interaction $U$ extends on the link between the resonant level and the neighboring lead sites. In all calculations we have considered the case of a half filled band and zero temperature, $E_{\mathrm{Fermi}}=0, T=0$.

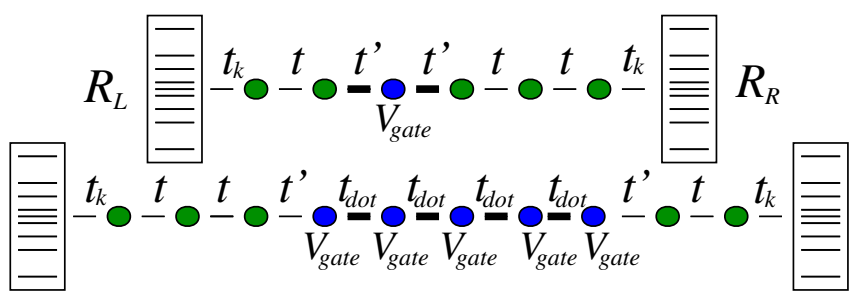

FIG. 1 (color online). Schematic representation of the calculational setup. Filled circles indicate the extended molecule, $e \mathcal{M}$. Gate voltage, $V_{\text {gate }}$, is applied to the molecule (dot), $\mathcal{M}$, only. Interacting sites are coupled by fat line links. Link labels indicate hopping amplitudes in the leads $t$, on the molecule $t_{\mathrm{dot}}$ and near the contacts $t^{\prime}$ and $t_{k}$. $\left(t=1, t_{\mathrm{dot}}=0.5\right.$ in all calculations presented.) $\mathcal{R}_{L}, \mathcal{R}_{R}$ denotes the reservoirs, here in $k$-space representation. Upper panel: single site resonance level model. Lower panel: five site model.
We calculate the GS of our model Eq. (2) by means of a DMRG calculation. DMRG [11,12] is a method that searches for an optimized subspace of the complete Hilbert space in which selected many body states can be described accurately. Notice, that our setup includes the leads in momentum space representation, $\mathcal{H}_{\mathcal{R}}$, which is nonstandard, but crucial for later transport calculations, Fig. 1; for details see [13]. In this way we obtain the local electron density on $e \mathcal{M}$ together with the occupation number of lead levels. Typically, we use more than 1300 states per block and ten finite lattice sweeps.

Next, we briefly explain how an exact DFT may be constructed generalizing earlier ideas by Gunnarsson and Schönhammer $[8,9]$. We define a KS-Hamiltonian $\mathcal{H}_{S}=$ $\mathcal{H}_{0}+V_{\mathrm{HXC}}$, with $\mathcal{H}_{0}=\mathcal{H}_{e} \mathcal{M}+\mathcal{H}_{\mathcal{R}}+H_{T}$ (free fermions) and an XC potential $V_{\mathrm{HXC}}=\sum_{j} v_{j} \hat{n}_{j}$ also including the Hartree term. Here, $n_{j}$ denotes the particle density. The sum is over the lattice sites $\ell$ of $e \mathcal{M}$ and the states $k$ of $\mathcal{R}$. According to theorems by Kohn and Hohenberg [14] and Kohn and Sham [15] a unique set of coefficients $v_{j}$ specifying the XC kernel exists, such that the KS-particle density coincides with the exact density of the many body GS. In practice, we find $v_{j}$ solving a standard optimization problem, which yields a final relative density mismatch of less than $10^{-10}$ per site.

Within DMRG the linear conductance can be obtained from evaluating the Kubo-formula [13,16]

$$
g=\frac{8 \pi e^{2}}{h}\left\langle\Psi_{0}\left|\hat{J}_{n_{1}} \frac{\eta\left(\mathcal{H}-\mathcal{E}_{0}\right)}{\left[\left(\mathcal{H}-E_{0}\right)^{2}+\eta^{2}\right]^{2}} \hat{J}_{n_{2}}\right| \Psi_{0}\right\rangle,
$$

where $\left|\Psi_{0}\right\rangle$ is the many body ground state, $\eta$ is the broadening parameter. $J_{n}$ is the current density operator at the bond between site $n$ and $n-1$ and $E_{0}$ denotes the GS energy. Because of particle number conservation, the dc conductance is independent of $n_{1}, n_{2}$. For the details of the procedure see Refs. [13,16].

The conductance $g$ - as in fact any dynamical correlator at $T=0$ - can be calculated evaluating proper GS matrix elements of certain known many body operators, see, e.g., Eq. (5). Therefore, the general principles of DFT apply and functionals exists, parameterized by $\eta$ (or frequency $\omega$, times $t, t^{\prime}$ etc.), which allow us to calculate such correlators from the GS density $n_{0}$ alone. The functional, which would yield the exact conductance $g\left[n_{0}\right]$ is not known. An approximate expression for $g$ yielding the exact KS conductance $g_{S}$ is obtained from Eq. (5) using the KS ground state with KS single particle energies $\epsilon_{p}$, the corresponding eigenstates for evaluating the matrix elements $J_{0 p}$ and $\mathcal{H} \rightarrow \mathcal{H}_{S}$

$$
g_{S}=\frac{8 \pi e^{2}}{h} \sum_{p, q} \frac{J_{0 p} J_{q 0} \eta\left(\epsilon_{p}-\epsilon_{q}\right)}{\left[\left(\epsilon_{p}-\epsilon_{q}\right)^{2}+\eta^{2}\right]^{2}} f\left(\epsilon_{q}\right)\left[1-f\left(\epsilon_{p}\right)\right] .
$$

$f(\epsilon)$ denotes the Fermi-Dirac occupation numbers.

We begin the report of our numerical results with the single level model (numerical parameters: $t^{\prime}=0.1$ ). This 


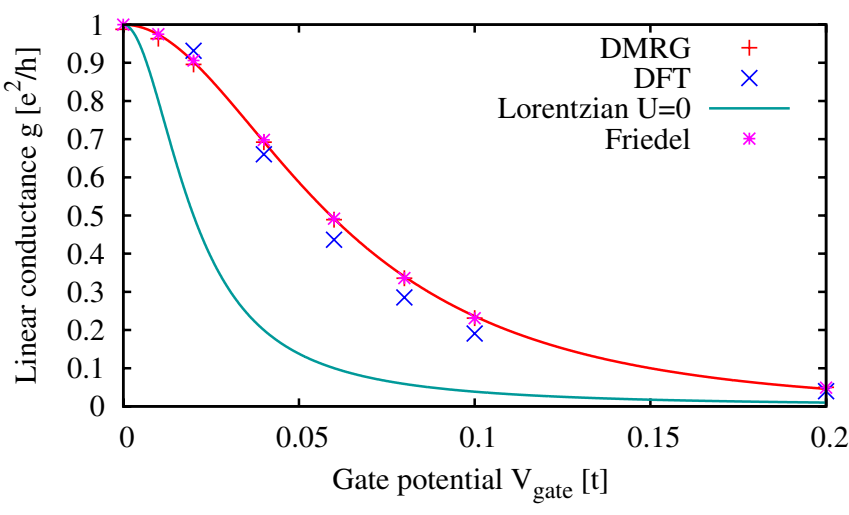

FIG. 2 (color online). Linear conductance over the gate voltage for the IRLM model with $t^{\prime}=0.1$. Comparison of conductance as calculated with three methods: exact analytical result (*, Friedel sum rule [17] and $n_{0}$ from DMRG), DMRG (+) and corresponding using the effective DFT Hamiltonian $(X)$. The line through the DMRG data is a guide to the eyes and the Lorentzian is the noninteracting result as reference. DMRG half width: $\Gamma=0.116 \pm 0.001$; DFT: $0.106 \pm 0.002$; noninteracting system, $\Gamma_{U=0}=0.04$.

model enjoys a particle-hole symmetry, so that the single transport resonance is pinned to the band center, $E=0$. Figure 2 shows the conductance, $g\left(V_{\text {gate }}\right)$, calculated analytically by Boulat and Saleur [17], the corresponding DMRG result and the Kohn-Sham conductance $g_{S}$. Comparison to the noninteracting limit $(U=0)$ exhibits a strong (280\%) interaction driven enhancement of the resonance width, $\Gamma=0.116$, compared to the noninteracting case $\Gamma=4 t^{\prime 2}=0.04$. DFT with exact XC, $g_{S}$, is able to reproduce this renormalization effect with accuracy better than $10 \%, \Gamma^{\mathrm{DFT}}=0.106$.

We now turn to the five site case, which affords four additional single levels that are not pinned to zero energy. Since now resonances experience an occupation dependent and interaction driven shift ("Coulomb blockade") with a corresponding change of the resonance width, this model can serve to investigate the DFT handling of such renormalization phenomena. Figure 3 displays the $g\left(V_{\text {gate }}\right)$ and $g_{S}\left(V_{\text {gate }}\right)$ conductance. Since particle-hole symmetry implies invariance under $V_{\text {gate }} \leftrightarrow-V_{\text {gate }}$, only the positive branch is shown. The first resonance at nonzero energy signalizes the transition, where the electron number $N_{\mathcal{M}}\left(V_{\text {gate }}\right)$ of the molecular dot changes between two and one, see Fig. 3. This happens at $V_{\text {gate }} \sim U-\Delta$, where $\Delta$ denotes the single particle level spacing. This expectation is roughly consistent with the numerical value 1.8 obtained from Fig. 3; for details see [16].

Figure 3 clearly shows that the DFT calculation perfectly well captures the position of the transport resonances. In addition, also the broadening of the resonance peaks is described reasonably well. Similar to the single level case, for the center peak width $10 \%$ deviations have to be accounted for. In contrast, a logarithmic plotting is required in order to make the relative deviations visible for

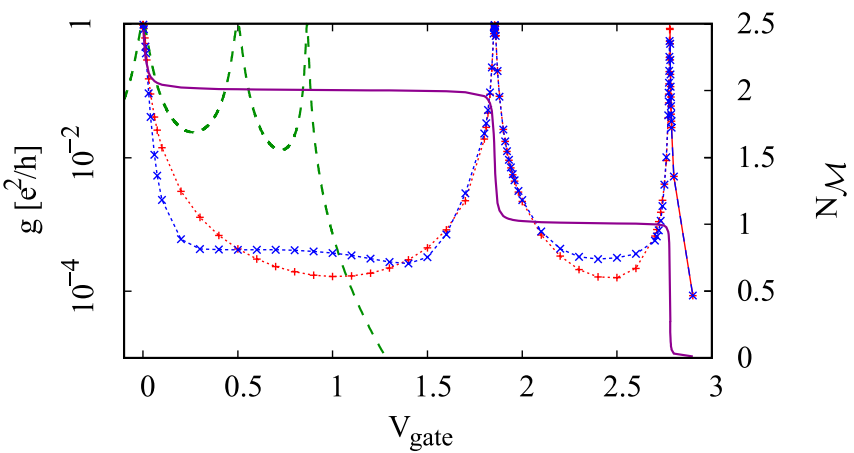

FIG. 3 (color online). Comparison of the exact conductance $(+$, dotted line as a guide) and the ground state DFT approximation $\left(\times\right.$, dashed line) for a five site system $\left(t^{\prime}=0.2, t_{\mathrm{dot}}=0.5\right.$, $U=2.0)$. For comparison the conductance of the noninteracting system $(U=0)$ is shown as well (long dashed line). The solid line indicates the particle number $N_{\mathcal{M}}\left(V_{\text {gate }}\right)$ of the molecule. The resonances of $g$ are situated at $V_{\text {gate }}=0,1.854$, and 2.779 with resonance widths of $\Gamma=0.026,0.015$, and 0.0033 .

the broadening of the shifted peaks. Remarkably, near resonances the variation of the conductivity is described over more than 3 orders of magnitude with deviations of a few percent or less.

In Fig. 4 we show the evolution of the local on site Hartree exchange correlation potential $v^{\mathrm{HXC}}$ with increasing gate voltage. The overall behavior is complicated, and a detailed discussion has to be relegated to Ref. [18]. Here, we can only briefly comment on two crucial aspects. First, $v^{\mathrm{HXC}}$ partially compensates $V_{\text {gate }}$ for repulsive voltages inbetween two resonances, keeping $N_{\mathcal{M}}$ integer cf. Fig. 3 . Second, at the resonance, $V_{\text {gate }} \approx 1.855$, the center peak of Fig. 4 rapidly decays. Thus, the double well structure in the full effective potential $v^{\mathrm{HXC}}+V_{\text {gate }}$ that was appropriate for two repulsive particles $N_{\mathcal{M}}=2$, transmutes into a single well hosting the lone particle, $N_{\mathcal{M}}=1$.

The predictive power of conductance calculations with ground state DFT may seem surprising at first sight because $V_{\mathrm{XC}}$ is neglected albeit it is very well known that the

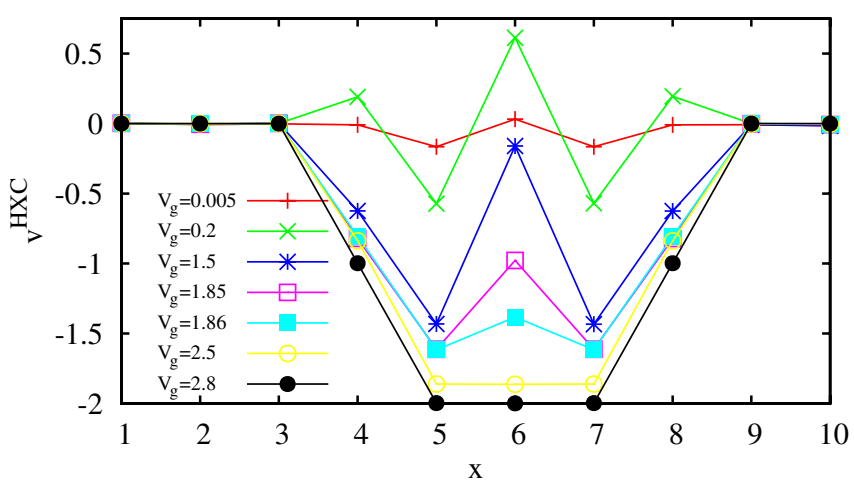

FIG. 4 (color online). Potential $v_{\ell}^{\mathrm{HXC}}$ on sites $\ell$ of $e \mathcal{M}$ corresponding to conductance data shown in previous Fig. 3 at $V_{\text {gate }}=$ $0.2,0.005,1.5,1.85,1.86,2.5,2.8$ (labeling center site, $x=6$, from top to bottom). 
bare KS-response yields incorrect excitation energies, which are shifted to proper values in TDDFT only by including dynamical correlations [19]. A closely related fact is that the bare KS-spectral function, $A_{S}\left(\omega, V_{\text {gate }}=0\right)$ exhibits excitation peaks at frequencies $\omega$ of the order of $\Delta$, and not $U$. The point that we wish to make here is that for correlated electron systems the dependence of $A_{S}$ on its arguments $\omega$ and $V_{\text {gate }}$ is quite different. The linear transport probes $A_{S}$ only in the vicinity of zero frequency. The evolution of $A_{S}\left(\omega=0, V_{\text {gate }}\right)$ with gate voltage is closely tied to the particle number and therefore can be physically meaningful and give quantitative results even if dynamical corrections are ignored.

We elaborate on this issue and give two reasons why in fact the good performance of $g_{S}$ is not entirely unexpected. (i) We are concerned with isolated resonances $\Gamma \ll \Delta, U$ which is a situation typical of the relatively small molecules that one deals with in the field of Molecular Electronics. These resonances occur precisely at the degeneracy point, where the $N$ and $N+1$ particle states of the molecular dot coincide in energy, so that the particle number is half integer $N+1 / 2$. Since the exact DFT monitors the true particle number on the dot, the degeneracy point of the KS-occupation and hence the KS-transport resonance coincide with the true value. (ii) It is much less obvious, why also the resonance width $\Gamma$ should be given very accurately, and in fact there is no reason to believe that this is always the case. However, under fairly general assumptions, one may argue that the width $\Im \Sigma$ of the transition in $N_{\mathcal{M}}\left(V_{\text {gate }}\right)$ gives a very good estimate for the width $\Gamma$ of the transport resonance. Indeed, consider a molecular dot invariant under exchange of left and right reservoirs (symmetric coupling). Then the single particle lifetime proper, $\Im \Sigma^{-1}$, of molecular excitations also appears as a transport rate $\Gamma \approx \Im \Sigma$, since the escape rates into the left and right leads, $\Gamma_{L}$ and $\Gamma_{R}$, simply coincide with $\Im \Sigma / 2$. Therefore $\Gamma^{-1}$ sets the (only) time scale for relaxation processes and hence it should also describe the width of transport resonances.

In summary, we have presented a method for performing exact DFT calculations for correlated electron systems coupled to external fermionic reservoirs. The approach is based on the DMRG. It has been used in order to calculate ground state Kohn-Sham conductances for the IRLM, which can be compared to exact results obtained with DMRG. We find that DFT calculations can describe positions and broadenings of transport resonances with a very good accuracy. It is thus suggested that the Kohn-Sham particles feel an effective voltage drop which does not deviate strongly from the physical voltage. In other words, dynamical corrections should be relatively small as long as vertex corrections can be ignored. This result is of particular importance for the field of Molecular Electronics. Namely, it nourishes hopes that conductance calculations based on the standard method should give much more quantitative results, once more accurate ground state functionals are available, so that a full time evolution of wave packets utilizing time dependent DFT may be evaded.

Useful discussions with K. Burke, F. Furche, G. Schneider, P. Schwab and P. Wölfle are greatfully acknowledged. This work was supported by the Center of Functional Nanostructures at Karlsruhe University. Part of the calculations have been performed at the XC2 of the SSC Karlsruhe under the grant number RT-DMRG.

[1] M. Brandbyge, J.-L. Mozos, P. Ordejon, J. Taylor, and K. Stokbro, Phys. Rev. B 65, 165401 (2002).

[2] F. Evers, F. Weigend, and M. Koentopp, Phys. Rev. B 69, 235411 (2004).

[3] A. Arnold, F. Weigend, and F. Evers, J. Chem. Phys. 126, 174101 (2007).

[4] M. Koentopp, K. Burke, and F. Evers, Phys. Rev. B 73, 121403(R) (2006); F. Evers and K. Burke, CRC Handbook on Molecular Electronics, edited by S. Lyshevski (CRC Press, Taylor \& Francis Group, 2007), Chap. 24.

[5] R. M. Dreizler and E. K. U. Gross, Density Functional Theory (Springer-Verlag, Berlin, 1990).

[6] C. Toher, A. Filippetti, S. Sanvito, and K. Burke, Phys. Rev. Lett. 95, 146402 (2005); S-H. Ke, H. U. Baranger, and W. Yang, arXiv:cond-mat/0609637v2.

[7] S. M. Lindsay and M. A. Ratner, Adv. Mater. 19, 23 (2007).

[8] O. Gunnarsson and K. Schönhammer, Phys. Rev. Lett. 56, 1968 (1986).

[9] K. Schönhammer, O. Gunnarsson, and R. M. Noack, Phys. Rev. B 52, 2504 (1995).

[10] N. Sai, M. Zwolak, G. Vignale, and M. Di Ventra, Phys. Rev. Lett. 94, 186810 (2005); Phys. Rev. Lett. 98, 259702 (2007); J. Jung, P. Bokes, and R. W. Godby, Phys. Rev. Lett. 98, 259701 (2007).

[11] S. R. White, Phys. Rev. Lett. 69, 2863 (1992); Phys. Rev. B 48, 10345 (1993).

[12] Density Matrix Renormalization-A New Numerical Method in Physics, edited by I. Peschel, X. Wang, M. Kaulke, and K. Hallberg (Springer, New York, 1999); R. M. Noack and S. R. Manmana, AIP Conf. Proc. 789, 93 (2005).

[13] D. Bohr and P. Schmitteckert, Phys. Rev. B 75, 241103(R) (2007).

[14] P. Hohenberg and W. Kohn, Phys. Rev. 136, B864 (1964).

[15] W. Kohn and L. J. Sham, Phys. Rev. 140, A1133 (1965).

[16] D. Bohr, P. Schmitteckert, and P. Wölfle, Europhys. Lett. 73, 246 (2006).

[17] E. Boulat and H. Saleur, Phys. Rev. B 77, 033409 (2008).

[18] P. Schmitteckert and F. Evers (to be published).

[19] G. Stefanucci, S. Kurth, E. K. U. Gross, and A. Rubio, in Molecular and Nano Electronics: Analysis, Design and Simulation, edited by J. Seminario, Elsevier Series on Theoretical and Computational Chemistry Vol. 17 (Elsevier, New York, 2007), p. 247. 